\title{
Molecular Docking, Toxicity and Antimicrobial Studies of P-Nitrobenzene Sulphonamide Bearing Leucine -Isoleucine Dipeptide Carboxamides.
}

\author{
Anya $\mathrm{Uzo}^{1}$, Okoro Christopher ${ }^{2}$, and Ike Christan ${ }^{3}$ \\ ${ }^{1}$ Project Development Institute \\ ${ }^{2}$ University of Nigeria Faculty of Physical Sciences \\ ${ }^{3}$ Enugu State University of Science and Technology Faculty of Applied Natural Sciences
}

June 4, 2020

\begin{abstract}
Dipeptide carboxamide bearing nitrobenzene sulphonamides offers new prospect towards the inhibition of hemoglobin degradation in erythrocytes by Plasmodium falciparum; a malaria parasite and glycosyl phosphatidyl inositol (GPI) pathway which controls the release of variable surface glycoprotein (VSG) from Trypanosoma brucei. Structural design carried out produced series of dipeptide carboxamide bearing p-nitrobenzene sulphonamide compounds formed by coupling isoleucine (Ileu) or leucine (Leu) bearing amine with p-nitrobenzene sulphonamide bearing Ileu or Leu. Variation of coupling sequence between amidated amino acids and p-nitrobenzene sulphonamide bearing amino acid generates a library of 52 amidated Leu-Ileu dipeptide carboxamides bearing p-nitrobenzene sulphonamide compounds. Antimicrobial properties were evaluated using in-silico molecular docking. Protein residues E.coli (coded: 5MMN), plasmepsin (coded: 3QSI), trypanosome brucei dihydrofolate reductase pyrimethamine (coded: 3QFX) and prostaglandin synthese (coded: 1EQG) were generated from protein data base and was used to study biological activities of the compounds on bacterial, malarial, trypanosomiasis and analgesic parasites respectively. Ofloxacin, celecoxib, chloroquine and melarsoprol were used for docking studies as reference drugs for antibacterial, analgesic, antimalarial and antitrypanosomiasis respectively. Selected compounds from docking studies were examined using ADMET to ascertain their toxicity profile. Results of the antimicrobial properties reveal that some of the compounds showed better binding affinity on plasmepsin and trypanosome brucei parasites compared to standard drug used as reference in the docking studies, making them a potential drug candidates for malaria and trypanosomiasis treatment.
\end{abstract}

\section{Introduction}

They are few reports on sulfa drugs bearing dipeptide, carboxamide and sulphonamide moiety in one molecule; thus, offering the prospects of developing new bioactive compounds. The rapid emergence of sulphonamide resistance organism is a challenge faced by pharmaceutical industry and has constantly prompted the need for researchers to develop new anti-microbial drugs use in treatment of infections caused by antibiotic resistant pathogens, as infections arisen from such microbial strains are potentially fatal [1].

Plasmodium, a parasite that causes malaria is one of the most widespread infectious diseases in the world with hundreds of millions affected and nearly two million people are killed each year, majority of them children [2]. Malaria parasite plasmodium falciparumattacks erythrocytes and feeds on its host's hemoglobin for nutrients necessary for its growth and development [3]. The inhibitory action of malaria drugs on Plasmodium falciparum is initiated by disrupting the vacuolar functions of the parasite [4]. Pep-statin A, a general inhibitor of aspartic proteases found in the initial steps of hemoglobin degradation process in erythrocytes [5-7], was reported to hinder hemoglobin degradation by extracting the digestive vacuoles 
of Plasmodium falciparum [8]. However, the emergence of drug resistant strains of Plasmodium falciparum was as a result of its ability to prevent vacuolar accumulation of weak bases [9].

Sleeping sickness also known as human African trypanosomiasis (HAT) is a vector-borne disease caused by two sub-species of protozoan parasites called T. brucei rhodesiense and T. brucei gambiense[10, 11]. These T. brucei parasites contain genes responsible for the synthesis of different types of variable surface glycoprotein (VSG) which eliminate the immune effect of the host's body, mainly mediated by antibodies [12,13]. The parasite uses soluble VSG as a strategy to modulate the host's immune response [14] and release same from the cell surface mediated by GPI hydrolysis mediated by phospholipase-C (PLC) and major surface proteases (MSP) found in the bloodstream form of T. brucei [15]. Antitrypanomiasis drug's potency is as a result of its inhibitory action on MSP and PLC enzymes which reduces the release of VSG molecules and these drugs binds effectively on the VSG sequence domain A and B [16].

Carboxamide derived from dipeptides are biocompatible and can effectively bind with most pathogenic organisms. The formation process of dipeptide allows infinite palette structure that is stable within living system and function without being coded [17]. Efforts are being made by researchers to develop a small molecule peptide based on self-assembling of nano and macro systems amino acids and has discovered that dipeptides could agglomerate into different nanostructures [18]. Depending on peptides design, they can agglomerate into different forms of supramolecular architectures such as monolayers, nano vesicles, ribbons and nanotubes, all in nanoscale dimensions [19, 20]; hence, having dipeptide as part of building block in drug synthesis is attractive as this will enhance drug lipophilicity, reduces particle size of drugs and generally improve drug efficacy.

\subsection{Methodology}

\subsection{Structural design of ligands}

A library of new fifty two (52) leucine-Isoleucine (Leu-Ileu) dipeptide carboxamide bearing $p$ - nitrobenzene sulphonamide compounds were designed and used as ligands in computational auto docking. The designed compounds has leucine, isoleucine and $p$-nitrobenzene sulphonamide as their skeletal backbone as seen in (figure 1). Each amino acid is coupled with $p$-nitrobenzene sulphonamide (figure 5) which saves as the first intermediates and was auto docked to ascertain it binding energy (Table 1). Second designed intermediates consist of amidated amino acid (Ileu-Leu) whose binding energy is shown in (Table 2 \& 3). Coupling of the designed intermediates produced a dipeptide carboxamide bearing $p$ - nitrobenzene sulphonamide compound. Using different amines for amino acid amidation and varying the coupling sequence between the amidated amino acids and $p$-nitrobenzene sulphonamide (figure 1) generated a library of fifty two (52) leucine-Isoleucine dipeptide carboxamide bearing $p$-nitrobenzene sulphonamide compounds. Among the amines used include; dimethylamine, diethylamine, piperidine, N-methylpiperazine, morpholine, pipecolic acid, pyrrolidine, 4-methylpiperidine, imidazole, oxazolidine, thiazolidine, 4, 5 - dihydro - $1 \mathrm{H}$ - imidazole and indole. ACD / Chem Sketch 2015 version and molecular builder modules in molecular operating environment (MOE) were used to draw the $2 \mathrm{D}$ structures of these ligands.

\subsection{Protein Preparation}

The 3D crystal structures of four (4) proteins were obtained from Protein Data Bank (PDB) (http://www.rcsb.org). Among them include;E.coli, gyrase (PDB code: 5MMN) for antibacteria,Plasmepsin I (PDB code: 3QS1) for anti-malaria,Prostaglandin $H_{2}$ synthase (PDB code: 1EQG) for analgesic and trypanosome brucei dihydrofolate reductase pyrimethamine (PDB coded: 3QFX) for antitrypanosomiasis. All the protein was then prepared using Molegro Virtual Docker (MVD).

\subsection{Molecular Docking Study}

The molecular docking studies were carried out to have a better knowledge on the designed compounds interaction at molecular level with pathogenic organisms using molecular operating environment (MOE) software program and Molegro Virtual Docker (MVD). The 3D and X-ray crystallographic structure of E.coli, DNA gyrase having (PDB code: 5MMN), Plasmepsin I (PDB code: 3QS1),Prostaglandin $H_{2}$ synthase (PDB 
code: 1EQG) andtrypanosome brucei dihydrofolate reductase pyrimethamine (PDB coded: 3QFX) were obtained from the Protein Data Bank (http://www.rcsb.org) and further modified for docking calculation. This study looks at molecular auto docking of the design compounds along with their intermediates for comparative studies of their binding affinity and reference drug on amino residues of the pathogens (Table 1-7). Structure of some intermediates and the 2D representation of their binding Interactions are shown (Figures 2-7).
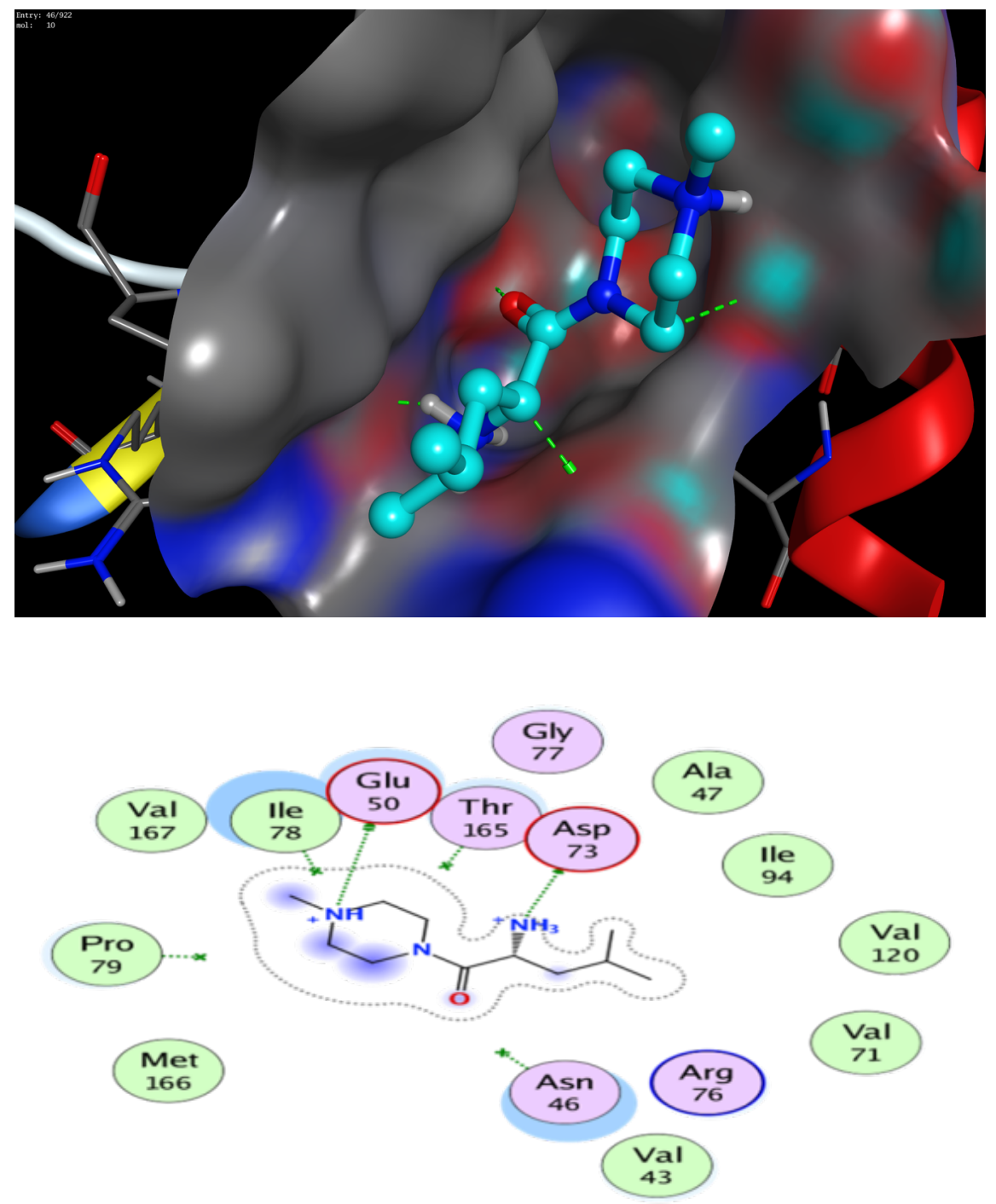

Figure 2: Binding Pose and 2D Representation of Binding Interaction between Compound 6D and Amino Residues of 5MMN 

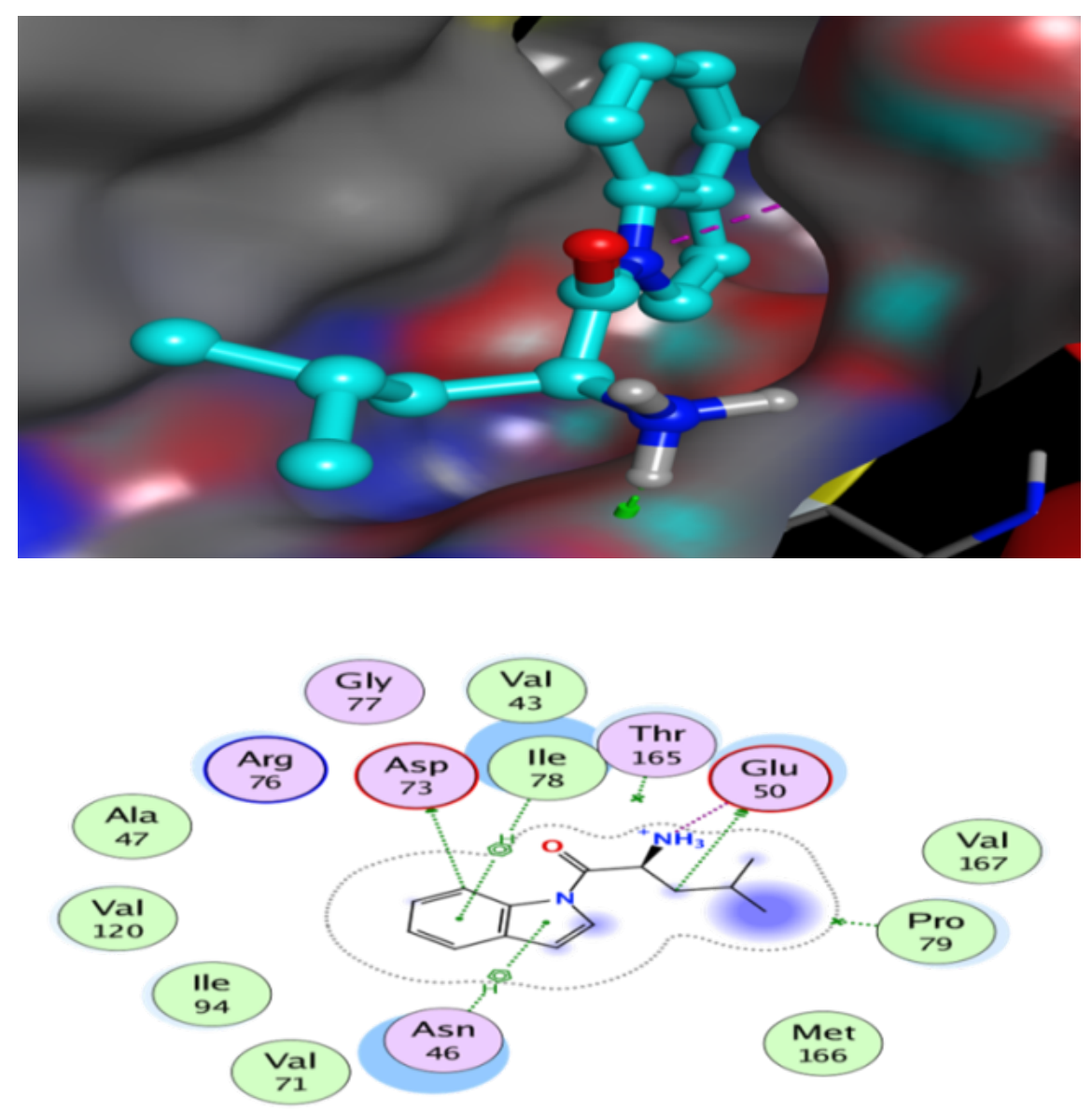

Figure 3: Binding pose and 2D representation of binding interaction between compound 15M and amino residues of $5 \mathrm{MMN}$ 

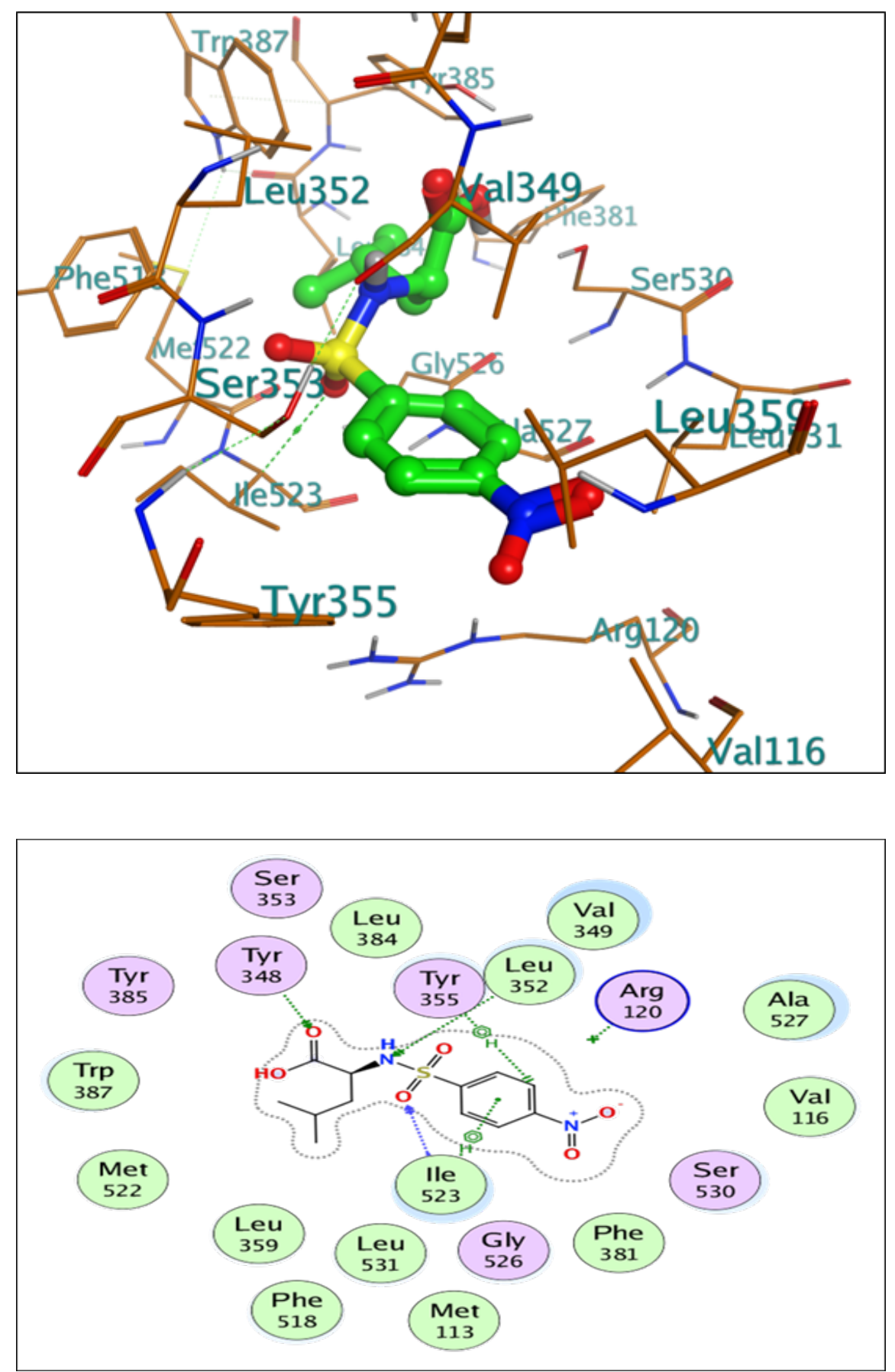

Figure 4: Binding pose and 2D representation of binding interaction between compound $\mathrm{Y}$ and amino residues of $1 \mathrm{EQG}$

Figure 5: P-Nitrobenzene Sulphonamide Bearing Leucine and Isoleucine intermediate Table 1; Binding free Energy in (kcal/mol) of $p$ - Nitrobenzene Sulphonamide Bearing Amino Acid 
Intermediates (compound 1 and 2)

\begin{tabular}{|c|c|c|c|c|c|c|}
\hline $\begin{array}{l}\text { Compound } \\
\text { ID }\end{array}$ & Amino Acid & $\begin{array}{l}\text { Receptor for } \\
\text { antibacterial } \\
(5 \mathrm{MMN})\end{array}$ & $\begin{array}{l}\text { Receptor for } \\
\text { analgesic } \\
\text { (1EQG }\end{array}$ & $\begin{array}{l}\text { Receptor for } \\
\text { analgesic } \\
\text { (1EQG }\end{array}$ & $\begin{array}{l}\text { Receptor for } \\
\text { antimalarial } \\
\text { (3QS1) }\end{array}$ & $\begin{array}{l}\text { Receptor for } \\
\text { antitry- } \\
\text { panosomiasis } \\
(3 Q F X)\end{array}$ \\
\hline $\mathrm{X}$ & Isoleucine & -9.94 & -11.08 & -11.08 & -10.87 & -12.18 \\
\hline Y & Leucine & -10.96 & -11.60 & -11.60 & -12.47 & -12.13 \\
\hline Native Ligand & Native Ligand & -11.50 & -11.50 & -11.34 & -11.28 & -9.72 \\
\hline Standard Drug & Standard Drug & -13.17 & -13.17 & -10.72 & -11.13 & -11.92 \\
\hline
\end{tabular}

Figure 6: Leucine Bearing Amine Intermediate

Table 2; Binding free Energy in ( $\mathrm{kcal} / \mathrm{mol})$ of Leucine Bearing Amine intermediates on Different Receptor

\begin{tabular}{|c|c|c|c|c|c|}
\hline Compound ID & Amine $\left(\mathbf{R}^{1}\right)$ & $\begin{array}{l}\text { Receptor for } \\
\text { antibacterial } \\
(5 \mathrm{MMN})\end{array}$ & $\begin{array}{l}\text { Receptor for } \\
\text { analgesic } \\
(1 \mathrm{EQG})\end{array}$ & $\begin{array}{l}\text { Receptor for } \\
\text { antimalarial } \\
(3 \mathrm{QS1})\end{array}$ & $\begin{array}{l}\text { Receptor for } \\
\text { antitrypanoso- } \\
\text { miasis } \\
(3 \mathrm{QFX})\end{array}$ \\
\hline $3 \mathrm{~A}$ & & -10.89 & -9.72 & -12.46 & -8.61 \\
\hline $4 \mathrm{~B}$ & & -10.00 & -9.36 & -12.57 & -9.44 \\
\hline $5 \mathrm{C}$ & & $-11.39-$ & -10.21 & -12.95 & -10.12 \\
\hline 6D & & -12.58 & -10.06 & -12.95 & -11.27 \\
\hline $7 E$ & & -10.90 & -10.90 & -12.90 & -9.87 \\
\hline $8 \mathrm{~F}$ & & -11.30 & -11.09 & -12.97 & -11.27 \\
\hline 9G & & -11.59 & -10.14 & -11.25 & -10.36 \\
\hline $10 \mathrm{H}$ & & -11.90 & -10.23 & -12.57 & -10.58 \\
\hline 11I & & -10.85 & -10.80 & -12.17 & -9.68 \\
\hline $12 \mathrm{~J}$ & & -10.84 & -9.77 & -11.91 & -10.08 \\
\hline $13 \mathrm{~K}$ & & $-10.82-$ & -10.39 & -12.02 & -8.98 \\
\hline $14 \mathrm{~L}$ & & -10.82 & -10.39 & -12.02 & -8.98 \\
\hline $15 \mathrm{M}$ & & -12.75 & -10.83 & -12.21 & -10.27 \\
\hline $\begin{array}{l}\text { Standard } \\
\text { drugs }\end{array}$ & $\begin{array}{l}\text { Standard } \\
\text { drugs }\end{array}$ & -13.13 & -10.72 & -11.13 & -11.92 \\
\hline
\end{tabular}

Figure 7: Isoleucine Bearing Amine

Table 3; Binding free Energy in ( $\mathrm{kcal} / \mathrm{mol})$ of Isoleucine Bearing Amine Intermediate on Different Receptors

\begin{tabular}{|c|c|c|c|c|c|c|c|c|c|}
\hline $\begin{array}{l}\text { Co1 } \\
\text { ID }\end{array}$ & $\begin{array}{c}\text { dCor } \\
\text { ID }\end{array}$ & $\begin{array}{l}\text { dAmine } \\
\left(\mathbf{R}^{1}\right)\end{array}$ & $\begin{array}{l}\text { Amine } \\
\left(\mathbf{R}^{1}\right)\end{array}$ & $\begin{array}{l}\text { Receptor } \\
\text { for } \\
\text { antibac- } \\
\text { terial } \\
(5 \mathrm{MMN})\end{array}$ & $\begin{array}{l}\text { Receptor } \\
\text { for anal- } \\
\text { gesic } \\
(1 \mathrm{EQG})\end{array}$ & $\begin{array}{l}\text { Receptor } \\
\text { for anal- } \\
\text { gesic } \\
\text { (1EQG) }\end{array}$ & $\begin{array}{l}\text { Receptor } \\
\text { for anti- } \\
\text { malarial } \\
(3 Q S 1)\end{array}$ & $\begin{array}{l}\text { Receptor } \\
\text { for anti- } \\
\text { malarial } \\
(3 Q S 1)\end{array}$ & $\begin{array}{l}\text { Receptor } \\
\text { for anti- } \\
\text { malarial } \\
(3 Q S 1)\end{array}$ \\
\hline $16 \mathrm{~N}$ & $16 \mathrm{~N}$ & & & -10.99 & -9.53 & -9.53 & -11.41 & -11.41 & -11.41 \\
\hline $17 \mathrm{O}$ & 170 & & & -10.02 & -9.87 & -9.87 & -11.92 & -11.92 & -11.92 \\
\hline
\end{tabular}




\begin{tabular}{|c|c|c|c|c|c|c|c|c|c|}
\hline $\begin{array}{l}\text { Compoun } \\
\text { ID }\end{array}$ & $\begin{array}{l}\text { dCompoun } \\
\text { ID }\end{array}$ & $\begin{array}{l}\text { dAmine } \\
\left(\mathbf{R}^{1}\right)\end{array}$ & $\begin{array}{l}\text { Amine } \\
\left(\mathbf{R}^{1}\right)\end{array}$ & $\begin{array}{l}\text { Receptor } \\
\text { for } \\
\text { antibac- } \\
\text { terial } \\
(5 \mathrm{MMN})\end{array}$ & $\begin{array}{l}\text { Receptor } \\
\text { for anal- } \\
\text { gesic } \\
\text { (1EQG) }\end{array}$ & $\begin{array}{l}\text { Receptor } \\
\text { for anal- } \\
\text { gesic } \\
\text { (1EQG) }\end{array}$ & $\begin{array}{l}\text { Receptor } \\
\text { for anti- } \\
\text { malarial } \\
(3 Q S 1)\end{array}$ & $\begin{array}{l}\text { Receptor } \\
\text { for anti- } \\
\text { malarial } \\
(3 Q S 1)\end{array}$ & $\begin{array}{l}\text { Receptor } \\
\text { for anti- } \\
\text { malarial } \\
(3 Q S 1)\end{array}$ \\
\hline $18 \mathrm{P}$ & $18 \mathrm{P}$ & & & -11.65 & -9.71 & -9.71 & -12.43 & -12.43 & -12.43 \\
\hline 19Q & $19 Q$ & & & -11.65 & -10.49 & -10.49 & -12.84 & -12.84 & -12.84 \\
\hline $20 R$ & $20 \mathrm{R}$ & & & -10.43 & -10.50 & -10.50 & -12.04 & -12.04 & -12.04 \\
\hline $21 S$ & $21 S$ & & & -10.81 & -10.36 & -10.36 & -12.23 & -12.23 & -12.23 \\
\hline $22 \mathrm{~T}$ & $22 \mathrm{~T}$ & & & -10.92 & -10.62 & -10.62 & -11.78 & -11.78 & -11.78 \\
\hline $23 \mathrm{U}$ & & & -10.92 & -10.92 & -10.92 & -9.45 & -12.05 & -12.05 & -12.05 \\
\hline $24 \mathrm{~V}$ & & & -11.35 & -11.35 & -11.35 & -9.75 & -11.97 & -11.97 & -11.97 \\
\hline $25 \mathrm{~W}$ & & & -10.86 & -10.86 & -10.86 & -10.08 & -11.70 & -11.70 & -11.70 \\
\hline $26 \mathrm{X}$ & & & -11.44 & -11.44 & -11.44 & -10.31 & -12.30 & -12.30 & -12.30 \\
\hline $27 Y$ & & & -11.63 & -11.63 & -11.63 & -9.89 & -11.98 & -11.98 & -11.98 \\
\hline $28 \mathrm{Z}$ & & & -11.59 & -11.59 & -11.59 & -10.66 & -12.34 & -12.34 & -12.34 \\
\hline $\begin{array}{l}\text { Standard } \\
\text { Drug }\end{array}$ & $\begin{array}{l}\text { Standard } \\
\text { Drug }\end{array}$ & $\begin{array}{l}\text { Standard } \\
\text { Drug }\end{array}$ & -13.13 & -13.13 & -10.72 & -10.72 & -10.72 & -11.13 & -11.92 \\
\hline
\end{tabular}

$P$ - nitrobenzene sulphonamide bearing leucine and isoleucine compounds is coupled with leucine and isoleucine bearing amine intermediates (figure $6 \& 7$ ) shown in (Table $2 \& 3$ ), this generate a series of leucine and isoleucine dipeptide carboxamide bearing $p$-nitrobenzene sulphonamide compounds (Table 4-7).

Reference drugs used in this study includes; ciprofloxacin for antibacterial, chloroquine for antimalarial, celecoxib for analgesic and melarsoprol for antitrypanosomiasis. The active sites was established from the binding pose and binding cavity of the crystal structure of each compounds with E.coli (PDB code: 5MMN), Plasmepsin I(PDB code: 3QS1), Prostaglandin $H_{2}$ synthase (PDB code: 1EQG) and trypanosome brucei dihydrofolate reductase pyrimethamine (PDB coded: 3QFX).

The best hit compounds were identified based on molecular interactions and binding pose with the active site components of the compound. Evaluation of binding energy and interactions of $p$ - nitrobenzene sulphonamide bearing dipeptide carboxamide compounds with pathogenic organism obtained from protein data base (PDB) revealed that compound ID 17 has the highest binding energy with $(-12.20 \mathrm{Kcal} / \mathrm{mol})$ onE.coli, DNA gyrase (PDB code: $5 \mathrm{MMN})$ active site which is lower than the reference drug with $(-13.17 \mathrm{Kcal} / \mathrm{mol})$. Compound ID 19, 34 and 52 recorded highest binding energy with $(-14.33 \mathrm{Kcal} / \mathrm{mol})$ inreductase pyrimethamine (PDB code: $3 \mathrm{QFX})$ active site, $(-10.09 \mathrm{Kcal} / \mathrm{mol})$ in Prostaglandin $H_{2}$ synthase (PDB code: $\left.1 \mathrm{EQG}\right)$ active site and $(-13.19 \mathrm{Kcal} / \mathrm{mol})$ in Plasmepsin I (PDB code: 3QS1) active site respectively. Compound 52 with binding energy of $(-13.19 \mathrm{Kcal} / \mathrm{mol})$ represented $18.5 \%$ increase in binding energy to the Plasmepsin I active site compared to chloroquine with binding energy of $(-11.13 \mathrm{Kcal} / \mathrm{mol})$, while compound 19 recorded an increase of $20.22 \%$ binding energy to trypanosome brucei dihydrofolate reductase pyrimethamine (PDB code: 3QFX) active site when compared with melarsoprol. Compound ID 8, 32 and 48 also showed higher binding affinity when compared with reference drug on trypanosome brucei dihydrofolate reductase pyrimethamine (PDB code: $3 \mathrm{QFX}$ ).

Table 4; Binding free Energy in (kcal/mol) when Coupling Sequence is (Ileu: Leu) to $p$ Nitrobenzene Sulphonamide as seen in compound (A) in figure 1 


\begin{tabular}{|c|c|c|c|c|c|c|c|}
\hline $\begin{array}{l}\text { Compound } \\
\text { NO. }\end{array}$ & $\begin{array}{l}\text { Amines ( } \\
\left.\mathbf{R}^{1}\right)\end{array}$ & $\begin{array}{l}\text { Receptor } \\
\text { for an- } \\
\text { tibacterial } \\
(5 \mathrm{MMN})\end{array}$ & $\begin{array}{l}\text { Receptor } \\
\text { for an- } \\
\text { tibacterial } \\
(5 \mathrm{MMN})\end{array}$ & $\begin{array}{l}\text { Receptor } \\
\text { for } \\
\text { analgesic } \\
\text { (1EQG) }\end{array}$ & $\begin{array}{l}\text { Receptor } \\
\text { for anti- } \\
\text { malarial } \\
(3 Q S 1)\end{array}$ & $\begin{array}{l}\text { Receptor } \\
\text { for anti- } \\
\text { malarial } \\
\text { (3QS1) }\end{array}$ & $\begin{array}{l}\text { Receptor } \\
\text { for antitry- } \\
\text { panosomi- } \\
\text { asis } \\
(3 Q F X)\end{array}$ \\
\hline 1 & & -10.04 & -10.04 & -8.25 & -11.09 & -11.09 & -11.50 \\
\hline 2 & & -9.56 & -9.56 & -7.85 & -10.59 & -10.59 & -11.43 \\
\hline 3 & & -9.70 & -9.70 & -7.00 & -11.49 & -11.49 & -11.88 \\
\hline 4 & & -11.95 & -11.95 & -7.55 & -12.23 & -12.23 & -12.05 \\
\hline 5 & & -11.44 & -11.44 & -7.74 & -12.28 & -12.28 & -11.14 \\
\hline 6 & & -10.14 & -10.14 & -6.97 & -13.00 & -13.00 & -11.26 \\
\hline 7 & & -9.59 & -9.59 & -6.95 & -12.45 & -12.45 & -11.24 \\
\hline 8 & & -10.49 & -10.49 & -8.03 & -11.70 & -11.70 & -12.31 \\
\hline 9 & & -9.69 & -9.69 & -8.28 & -11.99 & -11.99 & -11.48 \\
\hline 10 & & -10.35 & -10.35 & -7.42 & -10.84 & -10.84 & -11.61 \\
\hline 11 & & -9.77 & -9.08 & -9.08 & -12.27 & -12.27 & -11.19 \\
\hline 12 & & -10.08 & -7.37 & -7.37 & -11.49 & -11.49 & -11.19 \\
\hline 13 & & -10.04 & -8.80 & -8.80 & -11.01 & -11.01 & -11.57 \\
\hline Native & Native & -11.50 & -11.34 & -11.34 & -11.28 & -9.72 & -9.72 \\
\hline ligand & ligand & & & & & & \\
\hline $\begin{array}{l}\text { Standard } \\
\text { Drug }\end{array}$ & $\begin{array}{l}\text { Standard } \\
\text { Drug }\end{array}$ & -13.17 & -10.72 & -10.72 & -11.13 & -11.92 & -11.92 \\
\hline
\end{tabular}

Standard drug; Antibacterial = Ofloxacin; Analgesic $=$ Celecoxib

Antimalaria = Chloroquine; Antitrypanosomiasis = Melarsoprol

Table 5: Binding free Energy in $(\mathrm{kcal} / \mathrm{mol})$ when Coupling Sequence is (Ileu: Leu) to pNitrobenzene Sulphonamide as seen in compound (B) in figure 1

\begin{tabular}{|c|c|c|c|c|c|}
\hline Compound No & Amines $\left(\mathbf{R}^{1}\right)$ & $\begin{array}{l}\text { Receptor for } \\
\text { antibacterial } \\
(5 \mathrm{MMN})\end{array}$ & $\begin{array}{l}\text { Receptor for } \\
\text { analgesic } \\
\text { (1EQG) }\end{array}$ & $\begin{array}{l}\text { Receptor for } \\
\text { antimalarial } \\
\text { (3QS1) }\end{array}$ & $\begin{array}{l}\text { Receptor for } \\
\text { antitrypanoso- } \\
\text { miasis } \\
(3 Q F X)\end{array}$ \\
\hline 14 & & -9.77 & -8.69 & -11.27 & -11.64 \\
\hline 15 & & -9.11 & -8.61 & -11.61 & -11.64 \\
\hline 16 & & -10.46 & -7.74 & -11.53 & -11.18 \\
\hline 17 & & -12.20 & -9.11 & -11.25 & -11.91 \\
\hline 18 & & -9.59 & -7.31 & -11.28 & -11.44 \\
\hline 19 & & -10.43 & -7.17 & -11.83 & -14.33 \\
\hline 20 & & -9.92 & -7.06 & -11.38 & -11.89 \\
\hline 21 & & -9.73 & -7.36 & -11.08 & -10.72 \\
\hline 22 & & -9.49 & -8.55 & -11.16 & -11.97 \\
\hline 23 & & -9.48 & -7.97 & -11.81 & -11.98 \\
\hline 24 & & -9.97 & -8.26 & -11.45 & -11.44 \\
\hline 25 & & -10.20 & -9.80 & -11.81 & -12.14 \\
\hline 26 & & -11.31 & -7.00 & -10.86 & -11.15 \\
\hline Native ligand & Native ligand & -11.50 & -11.34 & -11.28 & -9.72 \\
\hline $\begin{array}{l}\text { Standard } \\
\text { Drug }\end{array}$ & $\begin{array}{l}\text { Standard } \\
\text { Drug }\end{array}$ & -13.17 & -10.72 & -11.13 & -11.92 \\
\hline
\end{tabular}


Standard drug; Antibacterial = Ofloxacin; Analgesic $=$ Celecoxib

Antimalaria = Chloroquine; Antitrypanosomiasis = Melarsoprol

Table 6: Binding free Energy in (kcal/mol) when Coupling Sequence is (Ileu: Leu) with $p$ Nitrobenzene Sulphonamide as seen in compound (C) in figure 1

\begin{tabular}{|c|c|c|c|c|c|c|c|}
\hline $\begin{array}{l}\text { Compound } \\
\text { No }\end{array}$ & $\begin{array}{l}\text { Amines } \\
\left(\mathbf{R}^{1}\right)\end{array}$ & $\begin{array}{l}\text { Amines } \\
\left(\mathbf{R}^{1}\right)\end{array}$ & $\begin{array}{l}\text { Receptor } \\
\text { for an- } \\
\text { tibacterial } \\
(5 \mathrm{MMN})\end{array}$ & $\begin{array}{l}\text { Receptor } \\
\text { for } \\
\text { analgesic } \\
\text { (1EQG) }\end{array}$ & $\begin{array}{l}\text { Receptor } \\
\text { for anti- } \\
\text { malarial } \\
(3 Q S 1)\end{array}$ & $\begin{array}{l}\text { Receptor } \\
\text { for antitry- } \\
\text { panosomi- } \\
\text { asis } \\
(3 Q F X)\end{array}$ & $\begin{array}{l}\text { Receptor } \\
\text { for antitry- } \\
\text { panosomi- } \\
\text { asis } \\
(3 Q F X)\end{array}$ \\
\hline 27 & & & -10.22 & -9.54 & -10.82 & -11.22 & -11.22 \\
\hline 28 & & & -10.14 & -7.99 & -10.72 & -11.23 & -11.23 \\
\hline 29 & & & -9.61 & -7.35 & -12.56 & -12.19 & -12.19 \\
\hline 30 & & & -10.46 & -8.82 & -12.60 & -12.17 & -12.17 \\
\hline 31 & & & -11.30 & -8.52 & -12.04 & -12.42 & -12.42 \\
\hline 32 & & & -10.26 & -9.20 & -11.79 & -13.23 & -13.23 \\
\hline 33 & & & -9.89 & -9.74 & -11.41 & -11.68 & -11.68 \\
\hline 34 & & & -10.31 & -10.09 & -10.60 & -12.11 & -12.11 \\
\hline 35 & & & 10.51 & -8.47 & -11.54 & -12.44 & -12.44 \\
\hline 36 & & & -10.90 & -9.01 & -11.95 & -12.64 & -12.64 \\
\hline 37 & & & -10.32 & -9.34 & -12.66 & -11.46 & -11.46 \\
\hline 38 & & & -9.81 & -7.33 & -11.68 & -11.99 & -11.99 \\
\hline 39 & & & -10.06 & -6.93 & -11.97 & -13.02 & -13.02 \\
\hline Native & Native & -11.50 & -11.50 & -11.34 & -11.28 & -11.28 & -9.72 \\
\hline ligand & ligand & & & & & & \\
\hline $\begin{array}{l}\text { Standard } \\
\text { Drug }\end{array}$ & $\begin{array}{l}\text { Standard } \\
\text { Drug }\end{array}$ & -13.17 & -13.17 & -10.72 & -11.13 & -11.13 & -11.92 \\
\hline
\end{tabular}

Standard drug; Antibacterial $=$ Ofloxacin; Analgesic $=$ Celecoxib

Antimalaria $=$ Chloroquine; Antitrypanosomiasis $=$ Melarsoprol

Table 7: Binding free Energy in (kcal/mol) when Coupling Sequence is (Ileu: Leu) to pNitrobenzene Sulphonamide as seen in compound (D) in figure 1

\begin{tabular}{|c|c|c|c|c|c|c|c|}
\hline $\begin{array}{l}\text { Compound } \\
\text { No }\end{array}$ & $\begin{array}{l}\text { Amines } \\
\left(\mathbf{R}^{1}\right)\end{array}$ & $\begin{array}{l}\text { Receptor } \\
\text { for an- } \\
\text { tibacterial } \\
(5 \mathrm{MMN})\end{array}$ & $\begin{array}{l}\text { Receptor } \\
\text { for } \\
\text { analgesic } \\
\text { (1EQG) }\end{array}$ & $\begin{array}{l}\text { Receptor } \\
\text { for } \\
\text { analgesic } \\
\text { (1EQG) }\end{array}$ & $\begin{array}{l}\text { Receptor } \\
\text { for anti- } \\
\text { malarial } \\
(3 Q S 1)\end{array}$ & $\begin{array}{l}\text { Receptor } \\
\text { for anti- } \\
\text { malarial } \\
(3 Q S 1)\end{array}$ & $\begin{array}{l}\text { Receptor } \\
\text { for antitry- } \\
\text { panosomi- } \\
\text { asis } \\
(3 Q F X)\end{array}$ \\
\hline 40 & & -10.03 & -8.26 & -8.26 & -11.40 & -11.40 & -12.50 \\
\hline 41 & & -9.54 & -7.71 & -7.71 & -11.49 & -11.49 & -10.68 \\
\hline 42 & & -10.72 & -7.16 & -7.16 & -11.12 & -11.12 & -11.55 \\
\hline 43 & & -10.64 & -7.52 & -7.52 & -11.42 & -11.42 & -11.42 \\
\hline 44 & & -11.03 & -7.66 & -7.66 & -13.06 & -13.06 & -12.54 \\
\hline 45 & & -11.01 & -6.77 & -6.77 & -11.44 & -11.44 & -12.70 \\
\hline 46 & & -9.59 & -7.27 & -7.27 & -11.34 & -11.34 & -11.61 \\
\hline 47 & & -9.88 & -5.77 & -5.77 & -12.99 & -12.99 & -11.98 \\
\hline 48 & & -10.90 & -9.02 & -9.02 & -11.57 & -11.57 & -13.35 \\
\hline
\end{tabular}




\begin{tabular}{|c|c|c|c|c|c|c|c|}
\hline $\begin{array}{l}\text { Compound } \\
\text { No }\end{array}$ & $\begin{array}{l}\text { Amines } \\
\left(\mathbf{R}^{1}\right)\end{array}$ & $\begin{array}{l}\text { Receptor } \\
\text { for an- } \\
\text { tibacterial } \\
(5 \mathrm{MMN})\end{array}$ & $\begin{array}{l}\text { Receptor } \\
\text { for } \\
\text { analgesic } \\
\text { (1EQG) }\end{array}$ & $\begin{array}{l}\text { Receptor } \\
\text { for } \\
\text { analgesic } \\
\text { (1EQG) }\end{array}$ & $\begin{array}{l}\text { Receptor } \\
\text { for anti- } \\
\text { malarial } \\
\text { (3QS1) }\end{array}$ & $\begin{array}{l}\text { Receptor } \\
\text { for anti- } \\
\text { malarial } \\
\text { (3QS1) }\end{array}$ & $\begin{array}{l}\text { Receptor } \\
\text { for antitry- } \\
\text { panosomi- } \\
\text { asis } \\
(3 Q F X)\end{array}$ \\
\hline 49 & & -10.25 & -7.62 & -7.62 & -11.40 & -11.40 & -12.11 \\
\hline 50 & & -9.82 & -7.66 & -7.66 & -11.67 & -11.67 & -12.42 \\
\hline 51 & & -10.30 & -8.10 & -8.10 & -11.57 & -11.57 & -11.48 \\
\hline 52 & & -9.89 & -7.55 & -7.55 & -13.19 & -13.19 & -12.89 \\
\hline Native & Native & -11.50 & -11.34 & -11.28 & -11.28 & -9.72 & -9.72 \\
\hline ligand & ligand & & & & & & \\
\hline $\begin{array}{l}\text { Standard } \\
\text { Drug }\end{array}$ & $\begin{array}{l}\text { Standard } \\
\text { Drug }\end{array}$ & -13.17 & -10.72 & -11.13 & -11.13 & -11.92 & -11.92 \\
\hline
\end{tabular}

Standard drug; Antibacterial $=$ Ofloxacin; Analgesic $=$ Celecoxib

Antimalaria $=$ Chloroquine; Antitrypanosomiasis $=$ Melarsoprol

2.4 Toxicity Study

\subsubsection{ADMET Descriptors}

The prediction of ADMET properties on drug candidate plays an important role in drug design as it account for the failure of most drugs in the clinical phases. ADMET descriptor was used to calculate and identify toxicity levels of a library of 52 compounds with characteristic pharmacokinetic properties [21]. Drug candidate with good toxicity and pharmacokinetic properties often pass test during clinical trial. The use of ADMET in prediction of drug properties before embarking on an expensive research or experimental is pertinent as it help in selecting successful drug candidate.

The present work used ADMET (absorption, distribution, metabolism, excretion, and toxicity) properties to evaluate the toxicity profile of the ligands. The procedure for pharmacokinetic involves using ADMET predictor model 9.5 which have the following parameters; gastrointestinal absorption (GI), blood-brainbarrier (BBB), P-glycoprotein (Pgp), cytochrome (1A2, 2C19, 2D6), Log P (lipophilicity), skin permeability $\log \mathrm{Kp}\left(\mathrm{cm}^{-\mathrm{s}}\right)$ and lead likeliness violation for predicting the properties compounds.

According to the model, a maximum cell permeability was achieved following the criteria of $\left(\mathrm{PSA}<140 \AA^{2}\right.$ and MiLog $\mathrm{P}<5)[22]$ and some of the designed compounds have shown a $\left(\mathrm{PSA}<140 \AA^{2}\right.$ and $\left.\operatorname{MiLog} \mathrm{P}<5\right)$ which satisfy the criteria. (MiLog P) and (Log P) predicts drugs lipophilicity and It has been reported that lipophilicity affects a number of pharmacokinetic parameters and high lipophilicity generally lower solubility, increased permeability in blood-brain barrier, gastrointestinal tract and other tissue membranes [22].

Low lipophilic prediction were observed in all the compounds and nine (9) of the compounds satisfied a $(\log \mathrm{P}>1$ or $<4)$ use for optimizing physicochemical ADME properties for oral drugs. The human gastrointestinal absorption (GI) of Ileu-Leu carboxamide bearing $p$-nitrobenzene sulphonamide compounds and their intermediates gave a range from (good absorption-high) to (low absorption-low), indicating good bioavailability that is responsible for drugs therapeutic effect (Table 8).

Blood brain barrier (BBB) penetration ranges from (low penetration-1) to (moderate penetration-2) with all the compounds indicating moderate penetration on BBB except for compound ID 28Z with low penetration. P-glycoprotein (Pgp) affinity with compounds varies from (affinity -1) to (no affinity -2) with only ten (10) of the designed compound showing no binding affinity to Pgp which denotes their non- inhibitory tendency to P-glycoprotein. While twenty one (21) of them has shown to bind with Pgp which help to achieve good bioavailability. Also, all the designed compounds were non-inhibitor to (CYP 450; 1A2, 2C19, 2D6) enzymes except for 10 (ten) compounds (Table 8). The toxicity profile depicts that all selected compound were 
inactive with respect to cytotoxicity, immunogenicity, androgen and estrogen receptors. While some of the designed compound were active to mutagenicity and carcinogenicity. Similarly, the hepatotoxicity levels of all compounds were satisfactory with exception to compound 13, 26 \& 39 (Table 10).

Table 8; Pharmacokinetics Profile of 31 Selected Compounds

Comp GI BBB Pgp CYP1A2 CYP2C19 CYP 2D6 Log P log Kp MiLog P TPSA Leadlikeness ID absorption permeant sub 12J High 2 No No No No $0.45-7.411 .8558 .691$ 13K High 2 No No No No $0.15-7.252 .2055 .561$ 14L High 2 No No No No

Table 9: Description of ADMET Parameters

\section{Name of ADMET Parameter Prediction level}

Gastrointestinal adsorption High (Good absorption) Low (Low absorption) Blood brain barrier (BBB) 1 ( low penetration)

\subsection{Toxicity Profile}

Acute oral toxicity test and alternative test protocols has been used to provide useful information about the acute toxicity of a compound [23]. Protox toxicity profile of 31 selected designed compounds revealed their acute toxicity tests using $\mathrm{LD}_{50}$ which defined the dose of these compounds that is lethal when $50 \%$ of animal are tested. Acute toxicity test result of 31 selected compounds revealed a LD50 range from (230 $-3800 \mathrm{mg} / \mathrm{kg}$ ). Furthermore, $\mathrm{LD}_{50}$ of $1000 \mathrm{mg} / \mathrm{kg}$ were recorded by 22 compounds, 7 compounds had $\mathrm{LD}_{50}$ $<1000 \mathrm{mg} / \mathrm{kg}$ while 2 compound gave $\mathrm{LD}_{50}$ of $3800 \mathrm{mg} / \mathrm{kg}$ (Table 10). All selected compounds exhibited good toxicity results on hepatotoxicity, immunogenicity, cytotoxicity, androgen and estrogen receptors. A total of 9 selected compounds were non- carcinogenic, while 10 of the compounds were a non- mutagenic.

Table 10: Protox Toxicity Profile of 31 Selected Compound

Compound $\mathrm{LD}_{50}$ Hepato- Carcino- Immuno- Muta- Cyto- Andragen Estrogen ID (mg/kg) toxicity genicity toxicity genicity 12J 3800 Inactive Inactive Inactive Inactive Inactive Inactive Inactive 13K 800 Inactive Inactive Inactive Inactive Inactive I

\section{Key: Active $=$ Interaction}

\section{Inactive $=$ No interaction}

\subsection{Result}

Computational auto-docking of the ligands provided essential information relating to the binding pose of $p$-nitrobenzene sulphonamide bearing leucine-isoleucine with different amines and their interaction with the active site of the protein residues codes: (5MMN, 1EQG, 3QSI, 3QFX). In this work, a library of 52 $p$-nitrobenzene sulphonamide bearing amidated leucine-isoleucine compounds was structurally designed. Compound ID 1-52 and 3A-28Z were the two intermediates designed and were coupled to generate 52 new compounds (Table 2-7). In silico auto docking of these 52 new compounds revealed their interaction and binding affinity with active site of the proteins which were compared with their intermediate and reference drugs.

Study of the docking activity revealed that known of the compound had better binding affinity to protein residues active sites ofE.coli, DNA gyrase (PDB: $5 \mathrm{MMN}$ ) and prostaglandin $H_{2}$ synthese (PDB: 1EQG) when compared to their reference drugs. Comparative analysis of binding affinity and interactions pattern of all the coupled compounds with their respective intermediates showed that an improved binding affinity was observed in all the coupled compounds compared to the intermediate in protein residue active site of 
trypanosoma brucei (PDB: 3QFX) (Table 4-7). Variation of coupling sequence between the intermediates gave compounds with improved binding energy and better toxicity results (Table 10).

Compounds ID 52 and 19 recorded the highest binding affinity with (-13.19) and (-14.33) on protein residues of Plasmepsin andtrypanosoma brucei respectively. This represents an increase in binding affinity of $18.5 \%$ and $20.12 \%$ in plasmepsin I (PDB: 3QSI) and trypanosoma brucei (PDB: 3QFX) active sites respectively when compared to reference drug (Tables $5 \& 7$ ).

\subsection{Discussion}

\subsection{Hemozoin interaction}

Hemozoin is a bio-crystal synthesized by plasmodium parasite which is responsible for hemoglobin proteolysis releasing free heme in the process. This free heme is toxic and can cause oxidative damage to parasite proteins, cell membranes and redox-active free heme (ferriprotoporphyrin IX [PPIXFe]). In order to avoid this toxicity, $P$ lasmodium parasites convert free heme into unreactive microcrystal made by the assemblage of heme dimers called malaria pigment or hemozoin [24]. The hemozoin pathway is unique for malarial parasite survival and offers an attractive target for the design of new anti-malarial drugs [25]. Chloroquine is known to be an efficient inhibitor of hemozoin formation [26], this is achieved by stacking with heme to form a stable $\pi-\pi$ complex bond, PPIXFe-chloroquine resulting to free heme entities that is not incorporated into hemozoin which could destroy the parasite. The result of docking studies on protein active site of plasmepsin (coded: 3QSI) revealed that most of the designed compounds exhibited higher binding affinity in contrast with standard chloroquine drug (Table $2-7$ ).

The binding affinity of compound ID 52 on protein active site ofplasmepsin is as a result of the 3rd oxygen atom from the nitro group $\left(\mathrm{NO}_{2}\right)$ in $p$-nitrobenzene sulphonamide moiety which formed two binding points interaction site with the residues of $\operatorname{Ser}^{35}$ and $\mathrm{Ala}^{36}(3.49 \AA$ and $3.23 \AA$ respectively). The two oxygen atom of $\mathrm{SO}_{2}$ moiety acts as a hydrogen bond acceptor with $\operatorname{Tyr}^{189}(2.67 \AA)$ and $\operatorname{Tyr}^{75}(3.26 \AA)$, this gives major interaction impact of $p$-nitrobenzene sulphonamide bearing leucine-isoleucine derivatives on plasmepsin protein thereby inhibiting it catalytic action. Also the aromatic ring of $p$-nitrobenzene sulphonamide formed two binding site points with $\pi$-cationic interactions of $\operatorname{Asn}^{74}(3.17 \AA)$ and $\operatorname{Met}^{73}(3.52 \AA)$ residues. Moreover, the $13^{\text {th }}$ nitrogen atom in sulphonamide moiety acted as a hydrogen bond acceptor with residue of Gly ${ }^{34}$ $(3.47 A)$. Hydrophobic interactions of the ligand with some amino acid residues were also revealed as shown in figure 8 .

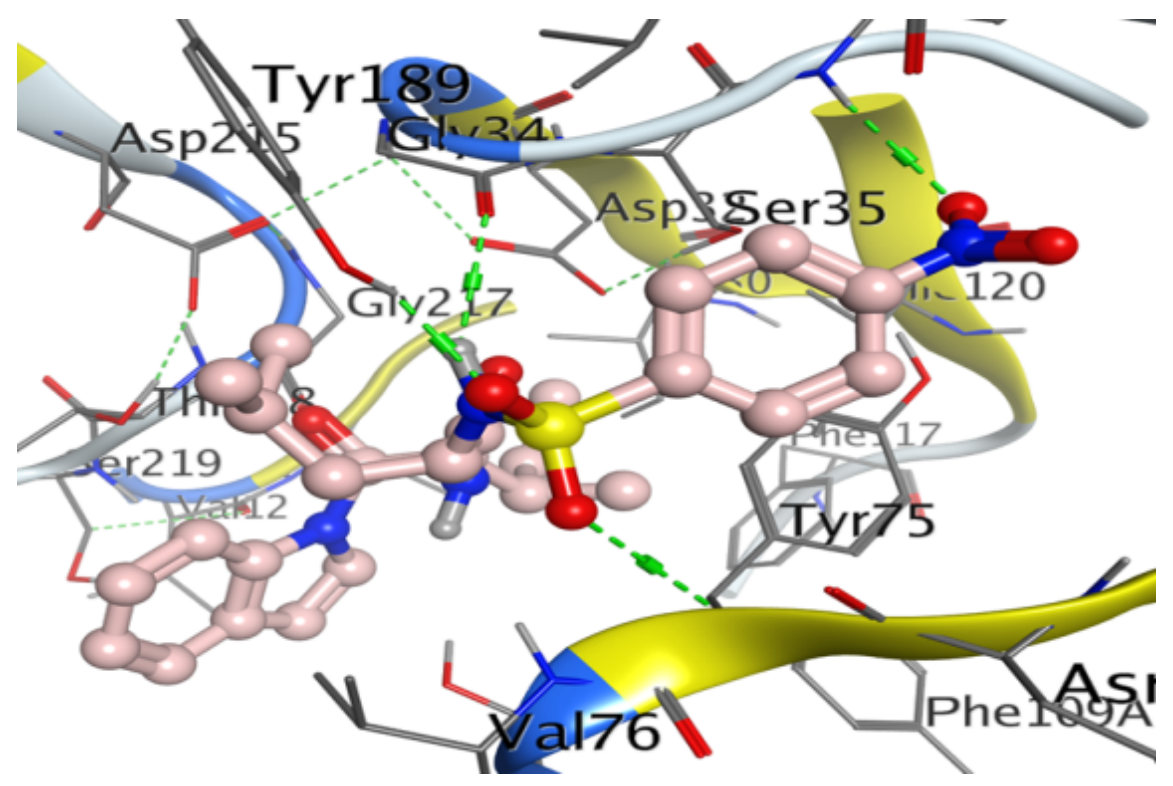




\section{Hosted file}

image72. emf available at https://authorea.com/users/329683/articles/456631-molecular-dockingtoxicity-and-antimicrobial-studies-of-p-nitrobenzene-sulphonamide-bearing-leucineisoleucine-dipeptide-carboxamides

Figure8: Binding pose and 2D representation of binding interactions between compound 52 and amino residue of plasmepsin I (PDB coded: 3QS1)

\subsection{Interaction of VSG molecules in T. brucei}

Trypanosoma brucei parasite is exclusively extracellular which escapes the host immune system by periodically changing the VSG molecule [27]; a strategy it uses to modulate the host's immune response. VSG are regularly release from $T$. brucei through proteolysis mediated by phospholipase-C (PLC) and major surface proteases (MSP) enzymes [28]. Some antitrypanomiasis drugs inhibit the release of VSG by targeting MSP and PLC enzymes in Trypanosoma brucei parasite. The interaction between the designed compounds and trypanosoma bruceiparasite (PDB coded: 3QFX) aided in identifying the antitrypanomiasis level of each compound. Docking studies shows that most of the designed leucine-Isoleucine amidated compound and p-nitrobenzene sulphonamide bearing dipeptide amine compound exhibited more binding affinity compared to melarsoprol drug use as reference (Table $2-7$ )

The interaction of compound ID 19 with protein residues oftrypanosome brucei ALA $^{34}(3.42 \AA, 3.18 \AA)$ and $\operatorname{VAL}^{33}(3.36 \AA)$ is from the $19^{\text {th }}$ and $20^{\text {th }}$ oxygen atom of $\mathrm{SO}_{2}$ moiety present in the compound, this shows that alanine interacted simultaneously with the two oxygen atoms to form two binding points using hydrogen bond acceptor as mode of interaction. Major interactions are on $13^{\text {th }}$ nitrogen atom from the first carboxamide group which interacts with residue of $\mathrm{Phe}^{58}(4.04 \AA), 6^{\text {th }}$ carbon atom of pipecolic acid with residue of $P$ he ${ }^{94}$ $(3.79 \AA), 16^{\text {th }} \& 30^{\text {th }}$ carbon atoms binding with $\operatorname{Ph}^{58}(4.49 \AA)$ and $\mathrm{Phe}^{58}(4.33 \AA)$ respectively in the protein residues of trypanosome brucei. The ability of these compounds to interact effectively with protein residues of trypanosome brucei gives rise to increase in binding affinity and making them potential drug candidates figure 9 .

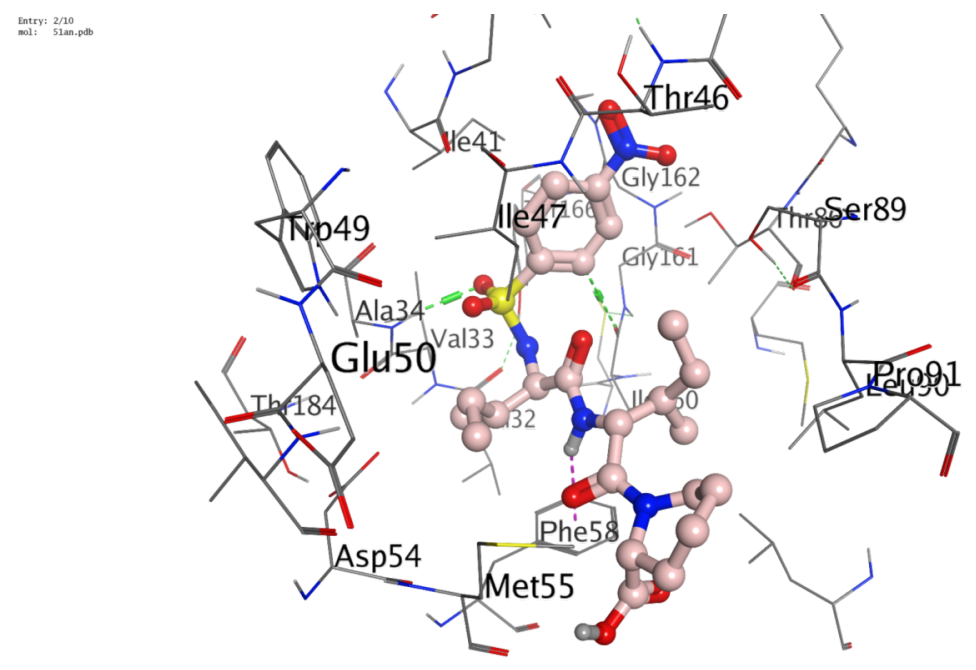

\section{Hosted file}

image74.emf available at https://authorea.com/users/329683/articles/456631-molecular-dockingtoxicity-and-antimicrobial-studies-of-p-nitrobenzene-sulphonamide-bearing-leucine- 
isoleucine-dipeptide-carboxamides

Figure 9: Binding pose and 2D representation of binding interactions between compound 19 and amino residue of trypanosome brucei (PDB coded: 3QFX)

\subsection{Conclusion}

A library of $52 p$-nitrobenzene sulphonamide dipeptide bearing leucine-isoleucine structural analogues and their respective intermediates where structurally designed and docked with E.coli(PDB code: 5MMN), Plasmepsin I (PDB code: 3QS1), Prostaglandin $\mathrm{H}_{2}$ synthase (PDB code: 1EQG) andtrypanosome brucei dihydrofolate reductase pyrimethamine (PDB coded: 3QFX). Docking study showed an increase binding affinity with the coupled compound when compared with their respective intermediates in protein residue active site of trypanosoma brucei (PDB: 3QFX). Result of ADMET revealed that some of the compound exhibited good pharmacokinetic properties and negligible toxicity. Coupling of the designed compound intermediates and varying the coupling sequence affected toxicity result which is in contrast with the intermediates and has proven to enhance binding affinity of the final compounds on protein active sites. This has resulted in giving out some of the best hit compounds in this work. Upon comparing the binding affinity of the reference drug with that of the compounds, higher binding affinity was recorded on plasmepsin and trypanosome brucei parasites by some of the compounds which can be screened and selected as potential drug candidates for malaria and trypanosomiasis treatment. Hence we conclude that the predicted parameter are exclusively used as basis for further design of dipeptide bearing benzene sulphonamide derivatives and understanding the effects of varying the coupling sequence on binding affinity and toxicity of the compound.

\section{Reference}

[1] C. Foucault,P. Brouqui, FEMS Immunology and Medical Microbiology (2007). Vol 49, Issue 2 pp- 173-313

[2] Sturchler, D. (1989) Parasitol. Today 5, 39-40.

[3] Scheibel, L. W. \& Sherman, I. W. (1988) in Malaria, eds. WernsdOH ofer, W. H. \& McGregor, I. (Churchill Livingstone, London), pp. 219-252.

[4] Olliaro, P. I. \& Goldberg, D. E. (1995) Parasitol. Today 11, 294-297.

[5] Francis, S. E., Gluzman, I. Y., Oksman, A., Knickerbocker, A.,Mueller, R., Bryant, M., Sherman, D. R., Russell, D. G. \& Goldberg, D. E. (1994) EMBO J. 13, 306-317.

[6] Gluzman, I. Y., Francis, S. E., Oksman, A., Smith, C. E., Duffin, K. L. \& Goldberg, D. E. (1994) J. Clin. Invest. 93, 1602-1608.

[7] Dame, J. B., Reddy, G. R., G., Yowell, C. A., Dunn, B. M., Kay, J. \& Berry, C. (1994) Mol. Biochem. Parasitol. 64, 177-190.

[8] Slater, A. F. G. (1993) Pharmacol. Ther. 57, 203-235.

[9] Umezawa, H., Aoyagi, T., Morishima, H., Matsuzaki, M.,Hamada, M. \& Takeuchi, T. (1970) J. Antibiot. (Tokyo) 23, 259-262.

[10] Ponte-Sucre A. An overview of Trypanosoma brucei infections: An intense host-parasite interaction. Front. Microbiol. 2016; 7:2126. doi: 10.3389/fmicb.2016.02126.

[11]Kristensson K., Nygård M., Bertini G., Bentivoglio M. African trypanosome infections of the nervous system: Parasite entry and effects on sleep and synaptic functions. Prog. Neurobiol. 2010;91:152. doi: 10.1016/j.pneurobio.2009.12.001.

[12] LaCount D.J., Gruszynski A.E., Grandgenett P.M., Bangs J.D., Donelson J.E. Expression and Function of the Trypanosoma brucei Major Surface Protease (GP63) Genes. J. Biol. Chem. 2003;278:24658-24664. doi: 10.1074/jbc.M301451200. 
[13] Baral T.N. Immunobiology of African trypanosomes: Need of alternative interventions. J. Biomed. Biotechnol. 2010; 2010:389153. doi: 10.1155/2010/389153.

[14] Grandgenett P.M., Otsu K., Wilson H.R., Wilson M.E., Donelson J.E. A function for a specific zinc metalloprotease of African trypanosomes. PLoS Pathog. 2007;3:1432. doi: 10.1371/journal.ppat.0030150.

[15] Deeks E.D. Fexinidazole: First Global Approval. Drugs. Springer International Publishing; Cham, Switzerland: 2019. pp. 1-6.

[16] riTryp DB: The Kineto plastid Genomics Resource. [(accessed on 9 March 2019)]; Available online: http://tritrypdb.org/tritrypdb/

[17] Winfree, E., Liu, F., Wenzler, L. A., \& Seeman, N. C. Design and self-assembly of two-dimensional DNA crystals. Nature (1998). 394, pp- 539-544.

[18] Reches, M., \& Gazit, E. (2003). Casting metal nanowires within discrete self-assembled peptide nanotubes. Science, 300, 625-7.

[19] Gazit, E. (2007). Self-assembled peptide nanostructures: the design of molecular building blocks and their technological utilization. Chem Soc Rev, 36, 1263-9.

[20] Vauthey, S., Santoso, S., Gong, H., Watson, N., \& Zhang, S. (2002). Molecular self-assembly of surfactantlike peptides to form nanotubes and nanovesicles. Proc Natl Acad Sci U S A, 99, 5355-60.

[21] Cumming J.G, Davis A.M, Muresen S, Haeberlein M, Chen H (2013). Chemical predictive modeling to improve compound quality. Nat Rev Drug Discovery 12:948-962

[22] S.Winiwarter, M.Ridderström, A.-L.Ungell, T.B.Andersson, I.Zamora (2007). Use of Molecular Descriptors for Absorption, Distribution, Metabolism, and Excretion Predictions; Comprehensive Medicinal Chemistry II Elsevier, vol 5, Pages 531-554.

[23] Litchfield, J.T., Jr., Wilcoxon, F. (1949) A simplified method of evaluating dose-effect experiments. J. Pharmacol. Exp. Therap. 96:113.

[24] singh .S, Srivastava . P (2015) Molecular docking studies of myricetin and its analogues against human PDK-1 kinase as candidate drugs for cancer. Comput Mol Biosci 5: 20-33

[25] Egan, T. J. 2002. Physico-chemical aspects of hemozoin (malaria pigment) structure and formation. J. Inorg. Biochem. 91:19-26.

[26] Christophe L, Joël L, Benoit-Vical F, Bernard M; (2007).Trioxaquines and Heme-Artemisinin Adducts Inhibit the In Vitro Formation of Hemozoin Better than Chloroquine. Antimicrobial Agents and Chemotherapy. 51 (10) pp 3768-3770; DOI: 10.1128/AAC.00239-07

[27] Vippagunta, S. R., A. Dorn, H. Matile, A. K. Bhattacharjee, J. M. Karle, W. Y. Ellis, R. G. Ridley, and J. L. Vennerstrom. 1999. Structural specificity of chloroquine-hematin binding related to inhibition of hematin polymerization and parasite growth. J. Med. Chem. 42:4630-4639

[28] Yao C., Donelson J.E., Wilson M.E. The major surface protease (MSP or GP63) of Leishmania sp. Biosynthesis, regulation of expression, and function. Mol. Biochem. Parasitol. 2003;132:1-16. doi: 10.1016/S01666851(03)00211-1.

\section{Hosted file}

PH.D PUBLICATION Anya 2.docx available at https://authorea.com/users/329683/articles/456631molecular-docking-toxicity-and-antimicrobial-studies-of-p-nitrobenzene-sulphonamidebearing-leucine-isoleucine-dipeptide-carboxamides 